\title{
Elektronische Verfahrensabwicklung von G2B-eServices: Vorgehensmodell und Anwendungs- fall zur Umsetzung der Dienstleistungsrichtlinie
}

\author{
Frank Hogrebe', Beate van Kempen ${ }^{1}$, Markus Nüttgens ${ }^{2}$ \\ ${ }^{1}$ Dezernat für Organisation, Personal, IT und Wirtschaftsförderung, \\ Burgplatz 1, 40213 Düsseldorf \\ ${ }^{2}$ Forschungsschwerpunkt Wirtschaftsinformatik, Universität Hamburg, \\ Von-Melle-Park 5, 20146 Hamburg \\ \{ frank.hogrebe|beate.vankempen\}@duesseldorf.de, \\ markus.nuettgens@wiso.uni-bamburg.de
}

\section{Ausgangslage und Motivation}

Vorgehensmodelle in der (Wirtschafts-)Informatik zielen auf eine durchgängige Beschreibung der Entwicklungs- und Einsatzphasen von Informationssystemen in Wirtschaft und Verwaltung. Sie bilden damit auch die Grundlage zur systematischen Realisierung elektronischer Verfahrensabwicklungen von unternehmensbezogenen Dienstleistungen in öffentlichen Verwaltungen (G2B-eServices). Prozesse dort haben bei abstrakter Betrachtung hinsichtlich ihrer Zielsetzung nur eine geringe strukturelle Varianz (i.d.R. Antragsbearbeitungen), lassen aber aufgrund der historisch gewachsenen Organisationsstrukturen, Verwaltungsvorschriften und Anwendungsfälle beträchtliche Unterschiede erkennen. Diese Heterogenität ist neben Fragen des Datenschutzes und der politischen Willensbildung ein wesentlicher Grund, warum es hier bisher kaum integrierte Anwendungssysteme gibt, sondern Insellösungen für einzelne organisatorische Bereiche.

Die Richtlinie 2006/123/EG des Europäischen Rates über Dienstleistungen im Binnenmarkt, kurz EU-Dienstleistungsrichtlinie (EU-DLR, 2006), fordert die öffentlichen Verwaltungen in den Mitgliedsstaaten auf, die erforderlichen rechtlichen, technischen und organisatorischen Voraussetzungen zu schaffen, die notwendig sind, um den Zielsetzungen der Richtlinie bis Ende 2009 nachzukommen. Durch die Richtlinie soll der freie Dienstleistungsverkehr innerhalb der Gemeinschaft für Unternehmen deutlich vereinfacht und erleichtert werden. Eine Kernforderung der Richtlinie ist die Bereitstellung unternehmensbezogener Verwaltungsleistungen in elektronischer Form (G2B-eServices). 
Integrierte Produkt- und Prozessmodelle (IPP) sind ein Ansatz, die Anforderungen der EU-Dienstleistungsrichtlinie an die öffentliche Verwaltung und die resultierende Komplexität modellbasiert abzubilden. Wesentliche Zielsetzung einer integrierten Produkt- und Prozessmodellierung ist ein gemeinsames Modellverständnis von Organisatoren und Modellierern auf der einen Seite und die Wiederverwendbarkeit von Produkt- und Prozessmodellen, bausteinbasierten Diensten und Softwarekomponenten auf der anderen Seite. Dies erfordert aber auch einen ganzheitlichen Ansatz im Systementwurf und einen neuen Grad der Zusammenarbeit in der IT, den Organisationsabteilungen und über die Organisationsbereiche hinweg bis zur Integration der Adressaten.

Ansätze domänespezifischer Vorgehensmodelle zu Teilbereichen der EUDienstleistungsrichtlinie finden sich bei (DIN FB 158 2009; DOL 2008; Algermissen und Instinsky 2008). Der aus Kundensicht relevante Phasenschwerpunkt zur technischen Realisierung der elektronischen Verfahrensabwicklung von G2B-eServices (EU-Kommission 2006, Art. 8) ist in Anwendungsfällen projektbezogen zu konkretisieren und zu validieren; solche Arbeiten sind bisher nicht publiziert. Der vorliegende Beitrag legt hierauf seinen Schwerpunkt und ist wie folgt aufgebaut:

Im zweiten Abschnitt werden die wesentlichen Anforderungsbereiche für die öffentliche Verwaltung zur Umsetzung der EU-DLR dargestellt und die Problemstellung bezogen auf die Anforderungen der elektronischen Verfahrensabwicklung konkretisiert. Ausgehend von einer Übersicht verwandter Arbeiten (Abschnitt 3) beschreibt der Beitrag ein Vorgehensmodell zur Umsetzung der EU-DLR für den Domänebereich der öffentlichen Verwaltung. Am Anwendungsfall einer deutschen Großstadt wird das Modell im Rahmen der Umsetzung der EU-DLR prototypisch angewandt (Abschnitt 4). Das Projekt wird methodisch und inhaltlich durch eine wissenschaftliche Begleitforschung abgesichert. Die Arbeit schließt mit einer Zusammenfassung und einem Ausblick auf den weiteren Forschungsbedarf.

\section{Anforderungen der EU-Dienstleistungsrichtlinie an die elektronische Verfahrensabwicklung von G2B-eServices}

Den Kern der Zielsetzungen der EU-Dienstleistungsrichtlinie bildet die Verwaltungsvereinfachung zugunsten von Unternehmen (Kapitel 2 der Richtlinie). Die EU-Kommission versteht unter dem Begriff der Dienstleistung ,jede selbständige wirtschaftliche Tätigkeit, die in der Regel gegen Entgelt erbracht wird“ (EUKommission 2007, S. 11). Von diesem auf die unternehmerische Tätigkeit bezogenen Dienstleistungsbegriff sind verwaltungsbezogene Tätigkeiten und Dienstleistungen abzugrenzen. Deren Zielsetzung ist es, die Unternehmensaktivitäten zu unterstützen, zu ordnen und damit die Aufnahme und Ausübung der unternehmerischen Tätigkeit zu gewährleisten. Hierzu zählen besonders Unterstützungsleistungen in Form der Bearbeitung von erforderlichen Genehmigungen und Regist- 
rierungen auf Basis von Rechtsnormen (wie Gesetze und Verordnungen). Auf diese unternehmensbezogenen Verwaltungsleistungen (G2B-eServices) fokussiert die elektronische Verfahrensabwicklung im Kontext der EU-DLR. Die Realisierung dieser elektronischen Verfahrensabwicklung stellt spezifische Anforderungen (A1-A4) an die Informationstechnik (EU-DLR 2006; EU-Kom. 2007; DOL 2008): Formularwesen (A1)

Zur Antragsbearbeitung von unternehmensbezogenen Verwaltungsleistungen auch „aus der Ferne“ (EU-Kommission 2006, Art. 8) sind notwendige Antragsformulare den Antragstellern elektronisch zur Verfügung zu stellen. Dies kann regelmäßig über Online-Portale der öffentlichen Verwaltungseinheit (im Frontoffice) erfolgen. Der Umfang kann dabei je nach Zuständigkeitsbreite einer Verwaltung mehrere Hundert Einzelformulare umfassen (KGSt 2008). Für eine medienbruchfreie Verfahrensbearbeitung sind die Formulardaten elektronisch an (weiter-)verarbeitende Systeme (im Backoffice) zu leiten. Um aus den Feldinhalten im Antragsformular strukturierte maschinenlesbare Austauschformate (wie XML) zu generieren, müssen die Formulare über entsprechende technische Funktionalitäten verfügen (BSI 2007, S. 23; DOL 2008, S. 122/128).

Authentifizierung und Identifikation (A2)

Zur rechtssicheren Kommunikation zwischen Kunden und der öffentlichen Verwaltung sind Identitätsdienste zur Authentisierung und Identifizierung der Akteure erforderlich (BSI 2007, S. 27; DOL 2008, S. 41). Nach Deutschem Recht ist der Absender eines elektronischen Dokumentes (z.B. Antragsteller) bisher nur dann eindeutig identifizierbar, wenn die Authentizität des Kommunikationspartners durch die Verwendung einer qualifizierten elektronischen Signatur nachgewiesen wird (DOL 2008 S. 113). Dabei erfolgt der Nachweis regelmäßig durch eine Abfrage der Identität bei sog. Trustcentern (BSI 2007, S. 15).

Verschlïsselungs- und Transportdienste (A3)

Für die übertragungssichere Kommunikation zwischen den Akteuren und einer geschützten Übermittlung von Daten bis ins Backend der Behörde sind geeignete Verschlüsselungs- und Transportdienste einzusetzen (DOL 2008 S. 109). Bei einer verschlüsselten Kommunikation tauschen die beiden Kommunikationspartner Dateien - sog. Schlüssel - aus (BSI 2007, S. 20). Jeder der beiden Kommunikationspartner verfügt über einen öffentlichen und einen privaten Schlüssel. Nur mit Hilfe beider Schlüssel kann eine verschlüsselte Datei entschlüsselt und damit lesbar gemacht werden.

Zablungswesen (A4)

Die vollständige elektronische Verfahrensabwicklung aus Kundensicht erfordert auch eine Funktionalität für Onlinezahlungen von Verwaltungsdienstleistungen (ePayment) (DOL 2008 S. 112). Der Kreis eines voll elektronischen Prozesses schließt sich mit der Möglichkeit, das erhaltene Produkt bzw. die Verwaltungsdienstleistung auch online bezahlen zu können. Onlinebezahlfunktionen ermöglichen dem Antragsteller die erforderlichen finanziellen Transaktionen über das Internet abwickeln zu können. Dabei wird der Bezug zur Verwaltungsdienstleis- 
tung über ein Akten- bzw. Kassenzeichen hergestellt. Über einen korrespondierenden Bezahllink können dem Antragsteller die für die Zuordnung vorgangsbezogenen Informationen bereits vorgegeben werden.

Ein Vorgehensmodell zur Umsetzung der elektronischen Verfahrensabwicklung im Sinne der EU-DLR (EU-Kommission 2006, Art. 8; DOL 2008, Kap. E) sollte die spezifischen Anforderungen an die Informationstechnik (A1-A4) mit einbeziehen.

\section{Verwandte Arbeiten}

Vorgehensmodelle unterscheiden sich durch eine durchgängige Beschreibung von Entwicklungs- und Einsatzphasen von Architektur-Konzepten und Methodologien, die den statisch-konstruktiven Charakter von Informationssystemen betonen und das Ziel verfolgen, ein ganzheitliches Rahmenwerk zur Beschreibung von Informationssystemen bereitzustellen (Nüttgens 1995, S. 2). Eine allgemeine Definition der Begrifflichkeit Vorgehensmodell (VM) hat sich bisher nicht etabliert. In Anlehnung an den Arbeitsbegriff des Arbeitskreises Vorgehensmodelle der Fachgruppe WI-VM der Gesellschaft für Informatik (AK-VMK 2007, S. 6) wird hier unter einem Vorgehensmodell „die Beschreibung eines arbeitsteiligen Prozesses mit Beteiligung mehrerer Rollen und Personen und deren Kombination von Methoden“ verstanden. Regelmäßige Anwendungsgebiete für Vorgehensmodelle liegen im Software Engineering (Höhn und Höppner 2008; Beck1999).

Die Phaseneinteilung hat das Ziel, eine komplexe Gesamtaufgabe in einzelne, einfacher beherrschbare Teilaufgaben zu zerlegen. Diese Vorgehensweise soll eine effiziente Planung, Steuerung und Überwachung der jeweiligen Phasenergebnisse und -übergänge ermöglichen. Die Abgrenzung der einzelnen Phasen ist zugleich Voraussetzung für die Festlegung der zeitlich-logischen Abfolge der einzelnen Aktivitäten. Tabelle 1 stellt wesentliche Vorgehensmodelle zum Geschäftsprozessmanagement und zur elektronischen Verfahrensabwicklung in der öffentlichen Verwaltung exemplarisch gegenüber; sortiert nach den Dimensionen Phasenabdeckung, Methodenunabhängigkeit und Domänenbezug, als für die Aufgabenstellung wesentliche Dimensionen (in Anlehnung an AK-VMK 2007, S. 7f.). Alternative Einteilungen finden sich bei Thomas et al. (2008), Fettke und Loos (2004). Die Übersicht dokumentiert die Pluralität in den sowohl wissenschaftlich- als auch praxisgetriebenen Ansätzen. Je komplexer ein Projekt ist, umso bedeutsamer ist es Kontrollabschnitte einzuführen und Zeitpunkte zu setzen, zu denen der Fortschritt, die Ergebnisse und Erkenntnisse bewertet werden (AK-VMK 2007, S. 23). Die Realisierung der elektronischen Verfahrensabwicklung von G2B-eServices im Kontext der EU-DLR stellt für den Domänenbereich der öffentlichen Verwaltung eine solche Komplexität dar; grundlegende Arbeiten hierzu finden sich bei Algermissen und Instinsky 2008; Deutschland Online 2008; Günther 2008; von Lucke et al. 2008. Die Literaturauswertung zeigt jedoch, dass bisher kein Vorgehensmodell zur elektronischen Verfahrensabwicklung von G2B-eServices zur Umsetzung der 
Anforderungen der EU-Dienstleistungsrichtlinie existiert. Insgesamt wurden 58 Vorgehensmodelle mit diesem Fokus ausgewertet.

\section{Vorgehensmodell zur Umsetzung der Anforderungen der EU-Dienstleistungsrichtlinie an G2B-eServices}

\subsection{Rahmenkonzept}

Die effektive Bereitstellung von Verwaltungsleistungen erfordert eine Verknüpfung der angebotenen Dienstleistungen und Dienstleistungsbündel (Produkte), der zur Verfahrensabwicklung eingesetzten Ressourcen (Prozesse) und der technischen Dienste und folglich eine Produkt- und Prozessarchitektur mit darauf ausgerichteten Anwendungssystemen und IT-Infrastruktur. Integrierte Produkt- und Prozessmodelle (IPP) sind ein Ansatz, die Anforderungen der EU-Dienstleistungsrichtlinie an die öffentliche Verwaltung und die resultierende Komplexität modellbasiert abzubilden (Hogrebe und Nüttgens 2008). Vor dem Hintergrund der effektiven Bereitstellung von Verwaltungsleistungen erhalten Konzepte einer Integrierten Produkt- und Prozessmodellierung im Vorgehensmodell besondere Beachtung.

Abbildung 1 zeigt das erarbeitete Vorgehensmodell im Überblick. Es umfasst vier Phasen mit jeweils korrespondierenden Teilaktivitäten. Das gewählte Modell ist das Resultat eines iterativen Entwicklungsprozesses eines 8-köpfigen Expertenteams bestehend aus drei Wirtschaftsinformatikern sowie fünf Experten aus dem Bereich der öffentlichen Verwaltung (davon drei IT-Spezialisten und zwei Organisatoren) basierend auf der Auswertung von 58 Vorgehensmodellen, Literatur und Praxisberichten.

Die Umsetzung der Anforderungen zur elektronischen Verfahrensabwicklung erfolgt hinsichtlich der Projektorganisation und der Zeit-, Aktivitäten- und Ressourcenplanung nach den gängigen Regeln zum Projektmanagement mit durchgängiger Dokumentation (Heckmann et al. 1998; DIN FB 158 2009):

Phase 1: Anforderungen und Zielsetzungen

Die Projektinitiierung repräsentiert den Projektstart und leitet die Aktivitäten des Vorgehensmodells ein. Im Rahmen der Voruntersuchung erfolgt zunächst eine Standortbestimmung und es werden die Rahmenbedingungen des Projektes (organisatorische, fiskalische, politische) eruiert und festgelegt. Kernanforderungen zur Umsetzung der elektronischen Verfahrensabwicklung gemäß der EU-DLR sind technikbezogen (Abschnitt 2) und somit als technische Basisfunktionalitäten und Dienste zu berücksichtigen. 
Tabelle 1: Verwandte Arbeiten

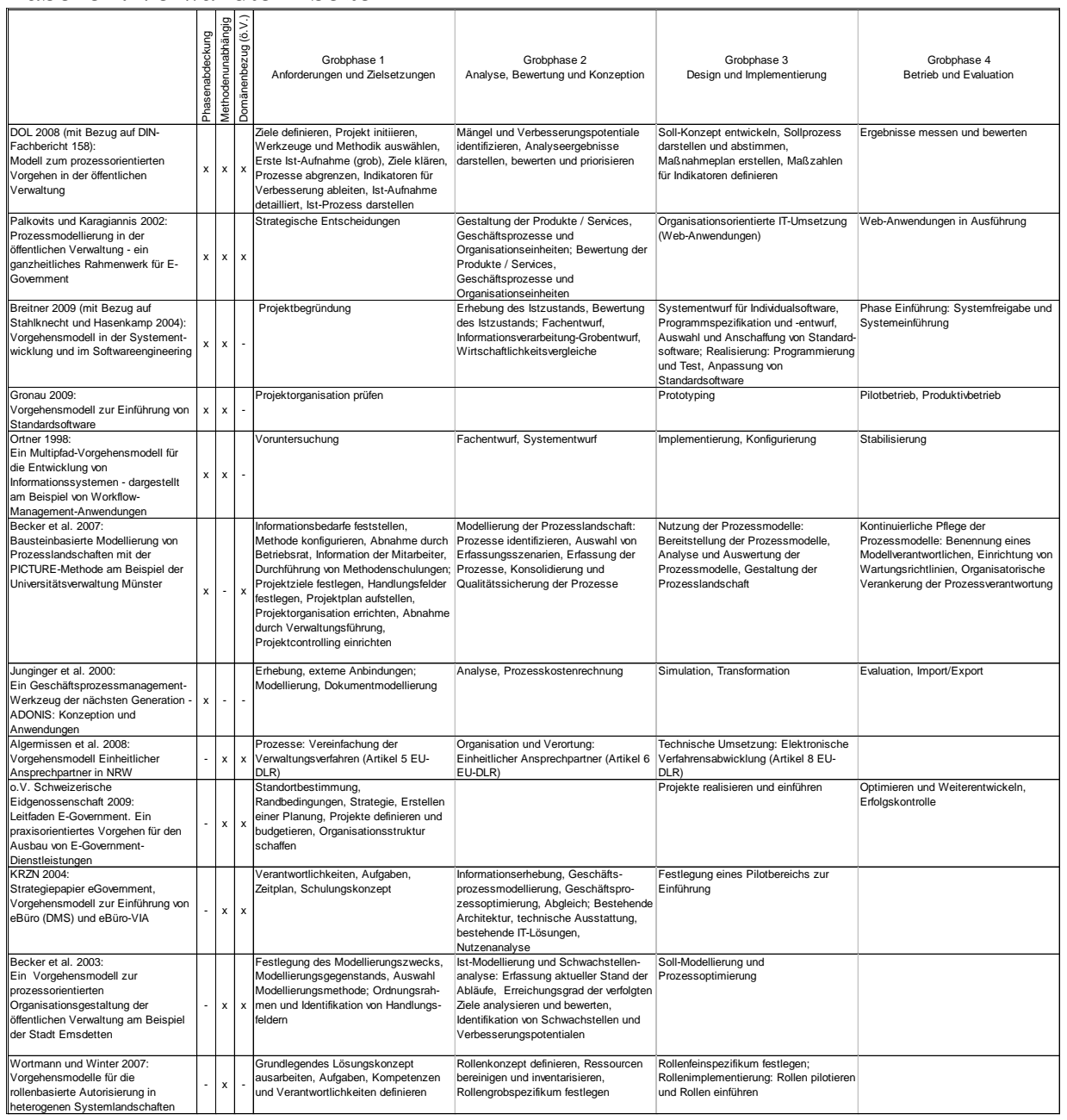

Die Ausprägung des Technisierungsgrades der elek-tronischen Verfahrensabwicklung (DOL 2008, Stufe 1, 1a oder 2) ist projektindividuell festzulegen; im Anwendungsfall wird die Stufe 2 realisiert. Den Anforderungen folgen die Festlegung der Zielsetzungen und die Phasenplanung.

Phase 2: Analyse, Bewertung und Konzeption

Im Rahmen der Analyse werden die unternehmensbezogenen Produkte und Prozesse identifiziert und dargestellt. Für die Ist-Modellierung bieten sich Beschreibungssprachen an, die durch Modellierungswerkzeuge technisch unterstützt werden. Aufgrund der im Regelfall geringeren Methodenkompetenz von Mitarbeitern in Fachbereichen sind für diesen Adressatenkreis leicht verständliche grafische Modelle hilfreich (Becker et al. 1999, S. 30). Die Bewertung der Prozesse im Rah- 
men einer Schwachstellenanalyse nimmt hierbei eine bedeutende Rolle ein, um Verbesserungspotentiale erkennen und nutzen zu können. Zur Umsetzung von Prozessoptimierungen bildet ein IPP-Rahmenkonzept die modelltheoretische Grundlage (Hogrebe und Nüttgens 2008). Weiterer wesentlicher Teil der Konzeption ist ein Schulungskonzept, damit notwendige Kompetenzen aufgebaut und Weiterentwicklungen frühzeitig kommuniziert und vorbereitet werden; bspw. bezogen auf die Verbesserung von Verwaltungsprozessen.

Phase 3: Design und Implementierung

Die Teilaktivitäten der Phase 3 repräsentieren den Wechsel von der Konzeptionierung und Modellierung hin zum DV-bezogenen Design der Prozessteile/-aktivitäten, die als Ergebnisse der Analyse- und Bewertungsphase als Projektzielsetzung zukünftig (teil-)automativ erfolgen sollen. Hier kommt der Auswahl servicebasierter Bausteine eine besondere Bedeutung zu, da das Ersetzen manueller Arbeitsschritte durch wieder verwendbare bausteinbasierte Dienste verschiedenste Optimierungs- und Synergiepotentiale bietet. So kann die durch einen SOA-Dienst frei gewordene Arbeitszeit bspw. in die Intensivierung der Bürger- und Unternehmensberatung eingesetzt werden. Die Implementierung umfasst die klassischen Teilschritte über das Prototyping bis hin zur technischen Abnahme.

\section{Phase 4: Betrieb und Evaluation}

Die Teilaktivitäten des Betriebs untergliedern sich in die Festlegung der dauerhaften Modell- und Prozessverantwortung, über den Pilotbetrieb bis hin zum Produktivbetrieb. Zur Überwachung des Betriebs bietet sich der Einsatz eines Betriebsund Evaluationsmonitorings an, um systematisch überprüfen zu können, ob und ggf. im welchem Maße die Zielsetzungen dauerhaft erreicht werden. Aktivitätenplanung zur Betriebsstabilisierung und Wartungsmodalitäten sind weitere Teilaspekte. Auch können die zugrunde liegenden Entscheidungsparameter (aus der Analyse- und Bewertungsphase) validiert und für Folgeprojekte konkretisiert und weiterentwickelt werden. In wieweit die Funktionalitäten und die Präsentation der Services (im Frontoffice) den Anforderungen der Zielgruppe entsprechen, sollte in Usability-Testings mit den Unternehmen/Bürgern evaluiert werden. Dies bietet weiteres Potential zur Optimierung und Weiterentwicklung der elektronischen Verfahrensabwicklung im Betrieb.

Je nach Rahmenbedingungen und individueller Projektziele kann sowohl die Reihefolge von Teilaktivitäten variieren als auch Teilschritte ganz entfallen. Die Phasenanordnung kann entweder streng sukzessiv aufgebaut sein, aber auch mehrere parallel auszuführende Phasenaktivitäten aufweisen (Thomas et al. 2008). 


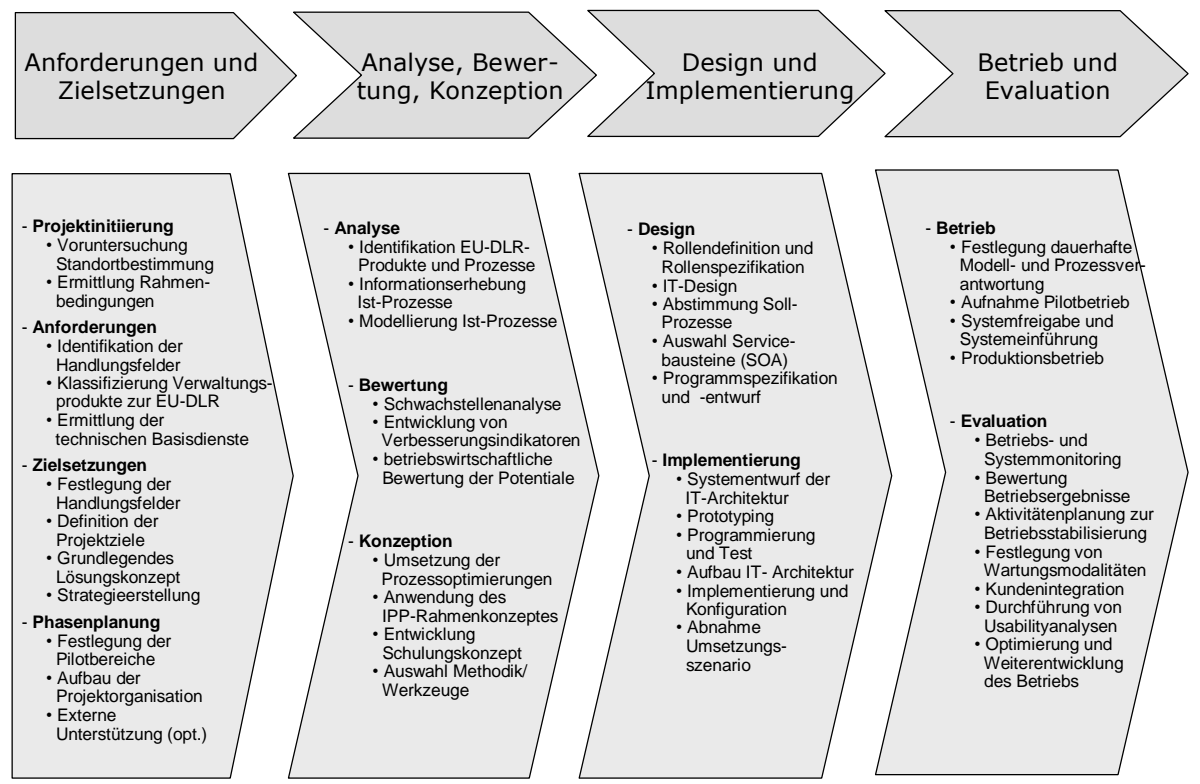

durchgehende Dokumentation und Projektmanagement-Aktivitäten

Abbildung 1: EU-DLR-Vorgehensmodell zur elektr. Verfahrensabwicklung

\subsection{Prototypische Anwendung des Vorgehensmodells}

Städte und Gemeinden sind in besonderem Maße gefordert, ihre Strukturen und Abläufe bis Ende 2009 auf die Dienstleistungsrichtlinie auszurichten, da sie die meisten Verfahrens- und Entscheidungszuständigkeiten des Staates in Deutschland auf sich vereinen (in Deutschland über 12.400 Städte und Gemeinden). Als Anwendungsfall wird daher eine deutsche Großstadt gewählt. In dieser haben rund 99\% aller Unternehmen weniger als 500 Beschäftigte und können, in Anwendung der Mittelstands(KMU)-Definition des Institutes für Marktforschung (2008), dem Mittelstand zugerechnet werden; dies sind im konkreten Fall rund 38.000 Unternehmen. Eine Einordnung nach der KMU-Definition der EU-Kommission (2003, für kleinere und mittlere Unternehmen), welche die Grenze bei 249 Beschäftigten zieht, verringert die Werte nur unwesentlich (im Nachkommabereich) und ist ohne Auswirkungen auf den gewählten Strategieansatz. Berücksichtigt man, dass mit abnehmender Unternehmensgröße die Umsetzung der EU-DLR an Bedeutung gewinnt, sind vorrangig Mittelstandsunternehmen die Zielgruppe. Die Anwendung des Vorgehensmodells führte im Rahmen des pilotierten Praxisprojektes zu den nachfolgenden Erkenntnissen (Ausschnitt):

Phase 1: Anforderungen und Zielsetzungen 
Die Projektinitiierung erfolgt im Anwendungsfall durch einen Beschluss der obersten Verwaltungsführung (Oberbürgermeister mit Beigeordnetenkollegium). Als Projektleiter wurde der für die Bereiche Organisation, IT, Personal und Wirtschaftsförderung zuständige Beigeordnete beauftragt. Grundlage dazu bildete eine Befragung von 360 Unternehmen, mit dem Ergebnis, dass rd. 63\% ein städtisches Unternehmensportal wünschen, von dem aus alle unternehmensbezogenen Dienstleistungen der Stadt online erreichbar sind. In dieses Portal war die elektronische Verfahrensabwicklung gemäß EU-DLR zu integrieren. Alle unternehmensrelevanten Dienstleistungen der Kommune wurden identifiziert und in einem EU-DLRProduktplan nach zuvor festgelegten Kriterien (wie Anzahl pro Jahr, Bedeutsamkeit für Unternehmen) klassifiziert. Die Bewertung und Klassifikation erfolgte in zwei Workshops des Expertenteams mit Unternehmern aus den Befragungen. Die vorhandenen und erforderlichen technischen Basisdienste wurden ermittelt, um den Kernprozess entsprechend der Zielsetzung zu realisieren. Handlungsfelder zur Anpassung an die IT-Architektur, Formularmanagementaspekte, Prozessoptimierung, Antragsstandverfolgung (Tracking) und Dialogfähigkeit über das Internet wurden festgelegt. Übergreifendes Ziel war, den in der EU-DLR vorgegebenen Termin (28.12.2009) zur Online-Abwicklung für die wichtigsten unternehmensbezogenen Verwaltungsverfahren zu erfüllen; im Anwendungsfall sind dies 41 unternehmensbezogene Dienstleistungen. Das Projekt wird durch eine phasenbezogene wissenschaftliche Begleitforschung abgesichert.

Phase 2: Analyse, Bewertung und Konzeption

Die Informationserhebung erfolgte in interdisziplinären Kleinteams mit den Fachbereichen, einem IT-Spezialisten und einem Organisationsmitarbeiter. Es wurden Teilschritte und Beteiligte des Prozesses identifiziert und dokumentiert. Auf dieser Grundlage wurden erste Rohentwürfe der Ist-Prozesse erstellt und die Prozessmodelle in Abstimmungsgesprächen ergänzt und verfeinert. Schwachstellen im Prozessablauf zur Gewerbemeldung wurden analysiert und Optimierungspotentiale identifiziert. Entsprechend dieser Potentiale wurde das Umsetzungskonzept entwickelt. Ein Schulungskonzept zur EU-DLR und zum Einsatz der Modellierungstechniken unterstützt die Umsetzung im Weiteren.

\section{Phase 3: Design und Implementierung}

Die IT-technischen Strukturen und Komponenten legen die Basis für das Design und die Implementierung. Ausgehend von funktional erweiterten OnlineFormularen, die entsprechend der Ergebnisse der Anforderungsanalyse neue Funktionalitäten, wie Sende-, Speicher-, sowie eine Signaturkomponenten, erhalten, werden die Daten in eine Datenstruktur für die Integration in die angeschlossenen Fachverfahren gebracht und unter Berücksichtigung der Datensicherheitsund -schutzrichtlinien verschlüsselt übertragen. Der sichere Rücktransport der Dienstleistung (z.B. die Bestätigung der Gewerbeanmeldung) kann dabei, unter Verwendung eines zentralen Verschlüsselungs- und Identifikations-Zertifikates, über einen Standard E-Mail-Client ohne weitere Hard- oder Software erfolgen. Das Konzept fußt auf einer integrativen „Mittlerschicht“, die es ermöglicht, auch alle 
weiteren Formulare auf diese prototypische Art (ggf. verbunden mit den unterschiedlichen Fachverfahren in den Ämtern) und eine gesicherte elektronische Kommunikation mit dem Dienstleistungserbringer bidirektional sicher zu stellen. Darüber hinaus wurde eine Online-Bezahlfunktion integriert. Der Kernprozess wurde gemäß Systementwurf und Aufbau der IT-Architektur prototypisch nach Programmierungs- und Testphasen sowie der Abnahme des konkreten Umsetzungsszenarios implementiert.

Phase 4: Betrieb und Evaluation

Für den Betrieb ist die Festlegung von dauerhaften Modell- und Prozessverantwortlichen unerlässlich, um eine Nachhaltigkeit in Pflege, Dokumentation und Weiterentwicklung sicher zu stellen. Im Anwendungsfall werden für jedes Prozessmodell zwei Personen (ein Vertreter) als Verantwortliche eingesetzt. Vor dem Pilotbetrieb wurden die Projektergebnisse mit den bereits einbezogenen Unternehmern getestet. Zur Evaluation wurden Usability-Testings durchgeführt (Pagel et al. 2009). Nach Aufnahme des Pilotbetriebes folgte die Systemfreigabe und einführung für den dauerhaften Produktivbetrieb durch die Verantwortlichen. Der Produktionsbetrieb unterliegt gleichermaßen einer ständigen Evaluierung, um den Betrieb zu stabilisieren und weiter zu entwickeln. Der geplante Projektzeitraum umfasste 20 Monate, abgeschlossen wurde das Projekt nach 14 Monaten.

\section{Zusammenfassung und weiterer Forschungsbedarf}

Der Beitrag beschreibt ein domänespezifisches Vorgehensmodell zur Umsetzung der elektronischen Verfahrensabwicklung zur EU-Dienstleistungsrichtlinie (EUDLR). Der aus Kundensicht relevante Phasenschwerpunkt zur technischen Realisierung der elektronischen Verfahrensabwicklung von G2B-eServices (EU-Kommission 2006, Art. 8) wird besonders fokussiert und an einem pilotierten Anwendungsfall prototypisch angewandt. Nach Diskussion der Anforderungsbereiche für öffentliche Verwaltungen zur Umsetzung der EU-DLR werden verwandte Arbeiten unter diesem Fokus untersucht. Das im Beitrag vorgeschlagene Vorgehensmodell basiert dabei auf Konzepten der integrierten Produkt- und Prozessmodellierung.

Am Anwendungsfall einer deutschen Großstadt wird das Modell im Rahmen der Umsetzung der EU-DLR angewandt. Die Erkenntnisse aus der prototypischen Anwendung des Vorgehensmodells sind vielversprechend. So konnte bspw. die elektronische Gewerbe-Anmeldung nach 14 Monaten produktiv gesetzt werden, der geplante Projektzeitraum umfasste 20 Monate. Gleichwohl ist dies die erste Anwendung des Modells, so dass Fragen zur Validität und Übertragbarkeit des Vorgehensmodells anhand von Folgeprojekten weiter zu untersuchen sind. Im nächsten Schritt dient das Vorgehensmodell als Grundlage zur Umsetzung der elektronischen Verfahrensabwicklung von weiteren unternehmensbezogenen Kerndienstleistungen der öffentlichen Verwaltung zur EU-Dienstleistungsrichtlinie. 


\section{Literatur}

Algermissen, L.; Instinsky, M.: Prozessmanagement für Verwaltungen als Grundlage verbesserter Dienstleistungen - Motivation einer prozessorientierten Umsetzung der EU-Dienstleistungsrichtlinie, in: Stember, J; Göbel, A. (Hrsg.): Verwaltungsmanagement für Unternehmen: Zwischen EU-Dienstleistungsrichtlinie, Bürokratieabbau und Wirtschaftsförderung. Münster 2008, 277 - 298.

Arbeitskreis Vorgehensmodelle: Katalog AK-VMK der Fachgruppe WI-VM der GI: Beschreibungskriterien für Vorgehensmodelle. Höhn, R. (Hrsg.), 2007.

Beck, K.: Extreme Programming Explained: Embracing change. Addison-Wesley, 1999.

Becker, J.; Schütte, R.; Geib, T.; Ibershoff, H.: Grundsätze ordnungsgemäßer Modellierung (GoM). Abschlussbericht, 1999. Online verfügbar unter: http://gvkplus/edok01.tib.uni-hannover.de/edoks/e001/303489413.pdf. Aufruf am 23.11.2009.

Bundesamtes für Sicherheit in der Informationstechnik (BSI): Handbuch eGovernment - Verschlüsseldung und Signatur, 2007.

https://www.bsi.bund.de/cae/servlet/contentblob/476808/publicationFile/2 8045/2_Krypto_pdf.pdf. Aufruf am 23.11.2009.

Deutschland-Online-Vorhaben. IT-Umsetzung der Europäischen Dienstleistungsrichtlinie. Projektbericht (Stand: 24.09.2008). http://www.deutschlandonline.de/DOL_Internet/broker.jsp?uMen=90040b06-56c2-3021-688d941ac0c2f214. Abruf am 23.11.2009.

DIN FB 158:2009-09. DIN-Fachbericht 158. Modell zum prozessorientierten Vorgehen in der öffentlichen Verwaltung. DIN Deutsches Institut für Normung, Beuth Verlag, Berlin, 2009.

EU-Dienstleistungstichtlinie: Europäisches Parlament und Europäischer Rat, Richtlinie 2006/123/EG über Dienstleistungen im Binnenmarkt vom 12.12.2006, in: Amtsblatt der Europäischen Union, L 376/36, Brüssel, 2006.

EU-Kommission der Europäischen Gemeinschaften: Handbuch zur Umsetzung der EU-Dienstleistungsrichtlinie. Amt für amtliche Veröffentlichungen der Europäischen Gemeinschaften (Hrsg.), Luxemburg, 2007.

EU-Kommission: Anhang zum Artikel 2 der Empfehlung der EU-Kommission vom 06.05.2003, Amtsblatt der EU, L 124/36, 2003.

Fettke, P.; Loos, P.: Systematische Erhebung von Referenzmodellen - Ergebnisse der Voruntersuchung. Information Systems \& Management (ISYM), Loos, P. (Hrsg.), Paper 19, Universität Mainz 2004. 
Günther, O.: Forschungsprojekt Prozess-Blaupause für die EU-Dienstleistungsrichtlinie, Institut für Wirtschaftsinformatik, Berlin, 2008.

http://www.prozessbibliothek.de/results.php?detail=fachprozesse. Abruf am 23.11.2009.

Heckmann, M.; Raether, C.; Nüttgens, M.: Werkzeugunterstützung im Service Engineering, in: Information Management \& Consulting, Sonderausgabe, 1998.

Höhn, R.; Höppner, S.: Das V-Modell XT. Anwendungen, Werkzeuge, Standards. eXamen.press, Springer, 2008.

Hogrebe, F.; Nüttgens, M.: Integrierte Produkt- und Prozessmodellierung: Rahmenkonzept und Anwendungsfall zur EU-Dienstleistungsrichtlinie, in: Loos, P.; Nüttgens, M.; Turowski, K.; Werth, D. (Hrsg.): MobIs 2008 - Modellierung betrieblicher Informationssysteme: Modellierung zwischen SOA und Compliance Management, Proceedings GI-Tagung, Saarbrücken, 2008.

Institut für Marktforschung (Hrsg.): Mittelstandsdefinition, 2008. http://www.ifmbonn.org/index.php?id=89. Aufruf am 23.11.2009.

Kommunale Gemeinschaftsstelle für Verwaltungsvereinfachung (KGSt): EUDienstleistungsrichtlinie: kommunale Leistungen mit Relevanz. Priorisierte Leistungslisten und Portfolioanalysen als Hilfestellung für die Praxis, KGStBericht 2/2008, Köln 2008.

Nüttgens, M.: Koordiniert-dezentrales Informationsmanagement: RahmenkonzeptKoordinationsmodelle - Werkzeug-Shell, Gabler-Verlag, Wiesbaden 1995.

Pagel, S.; Jürgens, A.; Nüttgens, M.; Adelskamp, P.; Hogrebe, F.; Lange, R.; van Kempen, B.: Usability-Analyse von Prozessmodellierungsmethoden. Abschlussbericht. Verbundprojekt der Fachhochschule Düsseldorf, Universität Hamburg, Landeshauptstadt Düsseldorf, Düsseldorf, 07/2009. [im Druck].

Thomas, O.; Leyking, K.; Scheid, M.: Vorgehensmodelle zur Entwicklung serviceorientierter Softwaresysteme. Thomas, O.; Leyking, K.; Scheid, M.: Vorgehensmodelle zur Entwicklung serviceorientierter Softwaresysteme. Institut für Wirtschaftsinformatik im DFKI, Universität des Saarlandes, Saarbrücken, 2008.

von Lucke, J.; Eckert, K.-P.; Breitenstrom, C.: EU-DLR-Einheitlicher Ansprechpartner. Rahmenarchitektur und technischer Lösungsansatz, 2008.

http://www.isprat.net/html/downloads/FOKUS-DLR-EAP-WhitePaper.pdf. Abruf am 23.11.2009. 